\title{
Sistema de Informação Geográfica para o Turista Deficiente: Análise de Publicações e Tecnologias
}

\author{
Geographic Information System Applied to Disable Tourist: \\ Systematic Review and Technologies
}

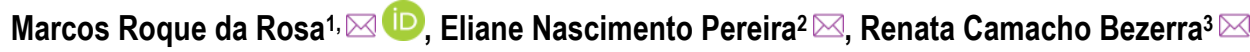 \\ 1 Universidade Estadual do Oeste do Paraná, marcosroquerosa@gmail.com \\ https://orcid.org/0000-0001-7145-1069 \\ Universidade Estadual do Oeste do Paraná, eliane.nascimentop@gmail.com \\ 3Universidade Estadual do Oeste do Paraná, renatacamachobezerra@gmail.com
}

Recebido (Received): 10/04/2019 Aceito (Accepted): 20/06/2019

\begin{abstract}
Resumo: O turismo acessível permite que as pessoas com deficiência atuem de forma independente e com dignidade em ambientes turísticos. Um Sistema de Informação Geográfica (SIG) permite a orientação do turista, fornecendo mapas digitais navegáveis, possibilitando a pesquisa e busca de informações de locais turísticos, bem como o conhecimento prévio da acessibilidade destes locais. A partir deste contexto, o artigo tem por objetivo apresentar uma análise de artigos científicos relacionados ao SIG para o turista deficiente e um levantamento de aplicativos existentes que ofereçam tal serviço. A pesquisa traz duas contribuições para este cenário: a primeira contribuição é a análise de como está sendo tratado o tema em relação à publicação de artigos científicos descrevendo as características dos trabalhos; a segunda contribuição é o levantamento de aplicativos existentes que podem auxiliar as pessoas com deficiência no turismo.
\end{abstract}

Palavras-chave: Turismo Acessível; Acessibilidade; Mobilidade; SIG

\begin{abstract}
Accessible tourism brings people with disabilities individual autonomy and independence in tourist environments. A Geographic Information System (GIS) provides tourist guidance by navigable digital maps and search about tourist places and advanced knowledge about accessibility of these places. This paper presents a systematic review related to Geographic Information System (GIS) applied to disabled tourist and applications that offer such service. The research offers two contributions to this scenario: the first analysis is how the topic is being related in scientific papers; the second contribution is to present the existing applications which can assist people with disabilities in tourism.
\end{abstract}

Keywords: Accessible Tourism; Accessibility; Mobility; GIS.

\section{Introdução}

Atualmente a população do Brasil conta com cerca de 45,6 milhões de brasileiros com algum tipo de deficiência, o que representa quase $24 \%$ de toda a população nacional (IBGE, 2010). Tendo como ponto de partida estes dados, tem-se como papel fundamental a construção de uma sociedade inclusiva, aberta e acessível, contando com a participação social e política de todos para o desenvolvimento de políticas públicas e formação humana.

Para promover a acessibilidade e possibilitar às pessoas com deficiência viver de forma independente e participar plenamente de todos os aspectos da vida, a sociedade como um todo deve tomar medidas apropriadas para assegurar às pessoas com deficiência o acesso, em igualdade de oportunidade com as demais pessoas, ao meio físico, ao transporte, à informação e comunicação, inclusive aos sistemas e tecnologias da informação e comunicação. Também se destaca que a sociedade deve ser consciente no papel e na responsabilidade de esforçar-se para a promoção e a observância dos direitos reconhecidos na Carta Internacional dos Direitos Humanos, ou seja, a colaboração de todos no respeito incondicional à dignidade humana (BRASIL, 2009). 
Pessoas com deficiência ou com mobilidade reduzida, tanto temporária como permanente, encontram obstáculos e barreiras à acessibilidade em edificações, transportes e mobiliários. Para eliminar estas barreiras e promover a inclusão, a tecnologia da informação está cada vez mais inserida no contexto de mobilidade urbana, com o acesso as informações pela internet, aplicativos móveis, equipamentos de localização por satélite. As tecnologias devem prover informações que auxiliem as pessoas com deficiência planejarem sua rotina para proporcionar mais conforto e segurança.

Segundo dados do Travel \& Tourism Economic Impact 2018 Brazil realizado pela World Travel \& Tourism Council (WTTC), a contribuição total das viagens e turismo para o Produto Interno Bruto (PIB) no Brasil foi de R $\$ 520,5$ bilhões, 7.9\% do PIB em 2017, e deverá crescer 2.5\% em 2018 e subir $2.8 \%$ ao ano para $\mathrm{R} \$ 701,7$ bilhões, 8,2\% do PIB em 2028. Em termos de empregos, diretos e indiretos, a contribuição total do setor de turismo para o emprego foi de $7.3 \%$ do emprego total, ou seja, 6.591 .500 postos de trabalho, com previsão de aumento de 1.9\% em 2018 (WTTC, 2018).

Prover informações e apoio à pessoa com deficiência ou com mobilidade reduzida, quando em viagem, para fins de entretenimento, atividades culturais e profissionais, e na atividade turística; permite a plena participação e inclusão destes na sociedade, igualdade de oportunidades e autonomia na utilização de serviços. Um Sistema de Informação Geográfica (SIG) aplicado ao turista com deficiência auxilia no planejamento da viagem, fornecendo conhecimento prévio de um local por meio de informações relacionadas, com a finalidade de organizar a locomoção e minimizar inconvenientes.

A partir deste contexto, este artigo tem por objetivo apresentar uma análise de artigos científicos relacionados a SIG para o turista deficiente e um levantamento de aplicativos existentes que ofereçam tal serviço. A presente pesquisa traz duas contribuições para este cenário: a primeira contribuição é a análise de como está sendo tratado o tema em relação à publicação de artigos científicos descrevendo as características dos trabalhos; a segunda contribuição é o levantamento de aplicativos existentes que podem auxiliar no turismo acessível.

Na próxima seção será apresentado um referencial sobre Turismo Acessível e Sistema de Informação Geográfica, a seção 3 descreverá o método adotado para a realização desta pesquisa, seguido dos procedimentos para o planejamento, identificação de estudo em bases de dados, a seleção e a extração. Na seção 4 serão apresentados os resultados separados em duas categorias: Categoria 1 - Tecnologias aplicadas ao turismo em geral, e Categoria 2 - Tecnologias aplicadas ao turista com deficiência. A seção 5 apresenta considerações finais, seguidas pela lista das referências utilizadas na pesquisa.

\section{Turismo Acessível e Sistema de Informação Geográfica}

O artigo 30 da Convenção Internacional sobre os Direitos das Pessoas com Deficiência reconhece o direito das pessoas com deficiência de participar na vida cultural e acesso a locais que ofereçam serviços culturais e turísticos (BRASIL, 2009). Este artigo da referida convenção coloca os operadores de turismo em alerta para alterar suas operações, com a finalidade de proporcionar o acesso a bens e serviços turísticos às pessoas com deficiência (DARCY; CAMERON; PEGG, 2010).

Os serviços turísticos, conforme o Código Mundial de Ética do Turismo (UNWTO, 2018), em seu artigo 7, devem abordar aspectos do turismo social, no âmbito de facilitar e encorajar o acesso ao lazer, a viagens e as férias, para famílias, jovens, estudantes, idosos e pessoas com deficiência. O desenvolvimento de infraestruturas, produtos e serviços turísticos acessíveis podem ter um efeito positivo nas operações comerciais, e que maior acessibilidade não só faz sentido financeiro, mas tem o potencial de promover objetivos sociais e ambientais (DARCY; CAMERON; PEGG, 2010).

A partir deste contexto, turismo acessível pode ser compreendido como:

O turismo acessível permite que as pessoas com requisitos de acesso, incluindo mobilidade, visão, audição e dimensões cognitivas de acesso, atuem de forma independente e com equidade e dignidade através da entrega de produtos, serviços e ambientes turísticos universalmente projetados. Essa definição inclui todas as pessoas, inclusive aquelas que viajam com crianças em carrinhos de bebê, pessoas com deficiências e idosos (DARCY; DICKSON, 2009, p 34, tradução nossa).

Segundo Darcy; Dickson (2009), quase todos nós, em algum momento de nossas vidas, poderemos ter uma deficiência, temporária ou permanente, resultado de um acidente ou uma lesão, acompanhar em viagem um familiar ou um amigo com requisitos de acesso. Sendo assim, acredita-se que o turista deficiente pode ser representado pela pessoa com requisitos de acesso em atividade turística, tanto em lazer ou a trabalho. 
Para apoiar o turismo acessível se faz presente a Tecnologia da Informação, na qual se destaca a utilização de um SIG, que permite a orientação do turista, fornecendo mapas digitais navegáveis, possibilitando a pesquisa e busca de informações de locais turísticos.

Compartilhando das ideias de Longley et al. (2013), "os sistemas de informação geográfica são uma classe especial de sistemas de informação que controlam não apenas eventos, atividades e coisas, mas também onde esses eventos, atividades e coisas acontecem ou existem".

Um SIG pode ser caracterizado pelos seguintes componentes (Figura 1), de acordo com Longley et al. (2013):

1) hardware do usuário, dispositivo pelo qual o usuário interage diretamente na realização de operações de SIG;

2) software que roda localmente na máquina do usuário;

3) banco de dados, que consiste em uma representação digital de aspectos selecionados de alguma área específica da superfície ou próxima da superfície da Terra;

4) procedimentos, um SIG requer gerenciamento, estabelecer processos, linhas de comunicação, com a finalidade de assegurar o SIG atinja suas necessidades;

5) as pessoas, na concepção, programação, manutenção, alimentação e interpretação dos dados;

6) a rede, permite a comunicação e compartilhamento de informação digital.

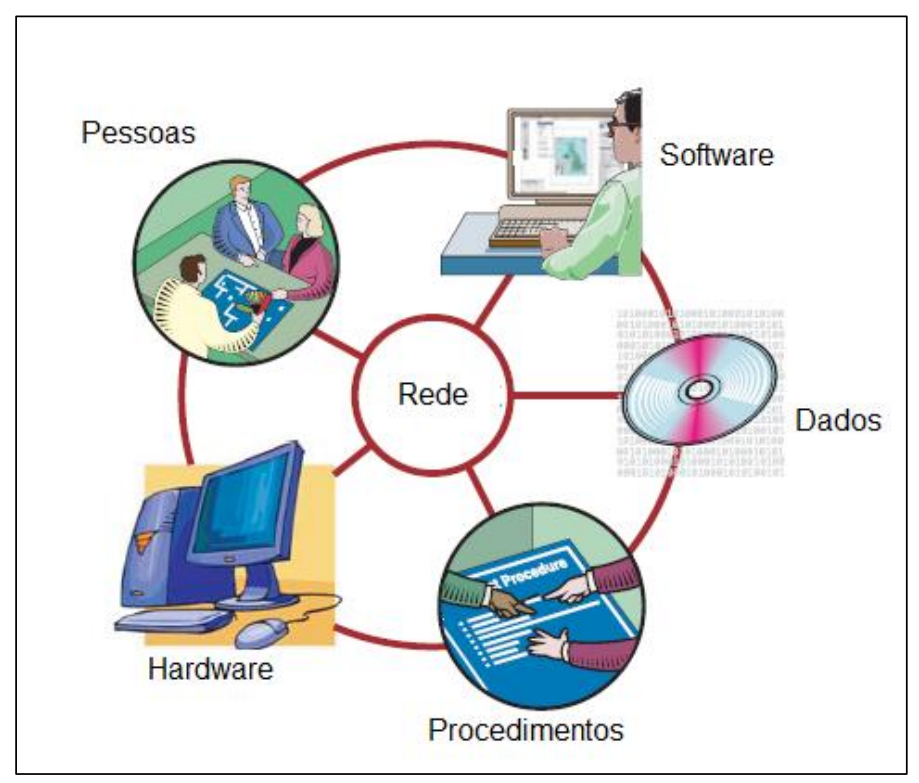

Fonte: (LONGLEY et al., 2013, p. 25)

Figura 1: Seis partes de um SIG

Problemas que envolvem um aspecto de localização, seja na informação utilizada para resolvê-lo, ou na solução propriamente dita, são denominados problemas geográficos, como exemplo, os viajantes e turistas, quando fornecem e recebem instruções de direção, selecionam hotéis em cidades não familiares e encontram seus próprios caminhos nos parques temáticos. Os SIG resolvem o antigo problema de combinar conhecimento geral com informação específica dando valor prático a ambos (LONGLEY et al., 2013).

\section{Método}

Este trabalho foi realizado em duas etapas: busca e análise sistemática de artigos relacionados ao tema da pesquisa. Para tanto, na busca de artigos utilizou-se de um protocolo com os seguintes itens: intervalo de anos das publicações, definição de bases de pesquisa específicas, uso de strings compostas para busca avançada dos artigos e explicitação de critérios de eliminação de artigos que não foram analisados.

Para desenvolver a análise sistemática dos artigos da pesquisa foram utilizados os procedimentos metodológicos tendo como base a revisão sistemática de literatura e a meta-análise, utilizando-se de práticas baseadas em evidências (TRANFIELD; DENYER; SMART, 2003). 
Para agregar legitimidade e a autoridade das evidências resultantes, as revisões sistemáticas podem fornecer aos profissionais e formuladores de políticas uma base confiável para formular decisões e agir. De acordo com Tranfield; Denyer; Smart (2003) a condução de uma revisão sistemática têm-se os seguintes estágios:

1) Planejamento da revisão: identificação da necessidade de uma revisão, preparação de uma proposta e desenvolvimento do protocolo;

2) Realização da revisão: identificação da pesquisa, seleção de estudos, avaliação da qualidade, extração de dados e síntese dos dados;

3) Reporte e divulgação: geração de um relatório e recomendações, colocação de evidências em práticas.

$\mathrm{Na}$ análise sistemática foi realizada a leitura integral dos textos, no qual são identificados os itens: introdução, os títulos das seções, tabelas e gráficos, definições de teoremas, conclusões e referências.

O período da busca foi de cinco anos, compreendendo os anos de 2013 a 2018, a pesquisa nas bases indexadas foi aplicada no dia 16 de julho de 2018. Foram selecionadas três bases de dados que indexam publicações científicas: IEEE, Web of Science e Science Direct. Essas bases indexam periódicos científicos de vários países, sendo consideradas pela comunidade acadêmica como bases relevantes para estudos relacionados à tecnologia da informação e também na área interdisciplinar.

As strings de buscas foram compostas com termos em inglês, possibilitando buscas abrangentes em meta-dados de título, resumo e palavras-chave: GIS, geographic information system, mobility, mobile, accessibility, acessible, disability, disabilities, disabled, tourism. Os artigos escritos em língua portuguesa também serão contemplados na busca, pois além dos meta-dados em português, apresentam título, abstract e keywords em língua inglesa. A partir da definição das palavras-chave, procurou-se utilizar de composições OR (ou), AND (e) e * (o asterisco no final serve como substituto para outras composições de palavras) para montar a string de busca; na sequência, tem-se uma Tabela $1 \mathrm{com}$ as bases, strings de buscas utilizadas e os resultados.

Foram adicionados cinco artigos obtidos por busca exploratória na ferramenta de busca Google Scholar, sendo caracterizados como entrada manual ao agrupamento geral, pois acredita-se que estes artigos podem enriquecer o trabalho, tratando de assuntos como: planejamento de rotas, desenvolvimento de aplicativos e serviços para pessoas com deficiência.

\begin{tabular}{|c|c|c|}
\hline BASE & STRING DE BUSCA & RESULTADO \\
\hline IEEE & 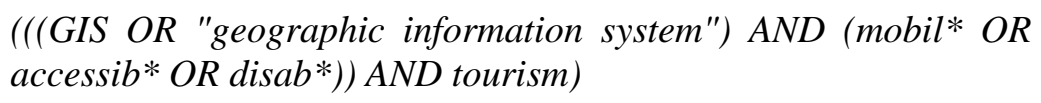 & 33 itens \\
\hline Web of Science & $\begin{array}{l}T S=((\text { GIS OR "geographic information system") AND (mobil } * \text { OR } \\
\left.\left.\text { accessib }^{*} \text { OR disab } *\right) \text { AND tourism }\right)\end{array}$ & 78 itens \\
\hline Science Direct & $\begin{array}{l}\text { pub-date }>2012 \text { and ((GIS OR "geographic information system") } \\
\text { AND (mobil* OR accessib* OR disab*) AND tourism)[All } \\
\text { Sources }(\text { Computer Science })] .\end{array}$ & 162 itens \\
\hline $\begin{array}{l}\text { Entrada Manual } \\
\text { TOTAL }\end{array}$ & Pesquisa exploratória & $\begin{array}{l}5 \text { artigos } \\
278 \text { itens }\end{array}$ \\
\hline
\end{tabular}

Para selecionar os artigos para leitura dos textos completos foram estabelecidos três principais critérios: 1) $O$ artigo deve ter relação direta com o tema SIG para o turista deficiente explicitado no título, resumo ou palavras-chave; 2) O documento deve ser classificado como artigo, sendo descartados livros e outros tipos; 3) O texto completo do artigo deve estar disponível para acesso.

Foram lidos os resumos e títulos dos 278 artigos identificados na etapa de busca sistemática, sendo descartados 2 artigos duplicados. Após a aplicação do critério de seleção, os artigos que não tem relação direta com o tema são descartados, e os documentos que não foram enquadrados como artigos foram descartados, restaram 18 artigos. Na busca do texto completo dos artigos nas bases pesquisadas, os 18 artigos foram encontrados com o texto completo para leitura. A partir disso, os 18 artigos formam selecionados para leitura integral e análise dos seus textos. 
No decorrer da busca e seleção, identificou-se a necessidade de categorizar em dois grupos os artigos, com a finalidade de facilitar a compreensão e enquadramento dos artigos, a saber:

Categoria 1 - Tecnologias aplicadas ao turismo em geral: nesta categoria estão os artigos que tratam tecnologias de apoio ao turismo em geral, pois não citam explicitamente ou diretamente a aplicação aos turistas com deficiência;

Categoria 2 - Tecnologias aplicadas ao turista com deficiência: nesta categoria estão os artigos que aplicam tecnologias para apoiar o turista com deficiência, mobilidade reduzida, idosos, grávidas, obesos, entre outros.

Após a leitura e análise dos artigos selecionados na revisão sistemática, procurou-se identificar os aplicativos relacionados à acessibilidade mais utilizados, efetuando buscas no serviço de distribuição digital de aplicativos Google Play Store. Foram encontrados dois aplicativos, com mais de 10.000 instalações, em julho de 2018, justificando o grande número de usuários. Estes aplicativos serão descritos na seção Resultados.

$\mathrm{Na}$ próxima seção deste artigo são apresentados os principais resultados obtidos a partir dos procedimentos descritos nesta seção de métodos e da leitura e análise dos artigos selecionados.

\section{Resultados}

Nesta seção serão apresentados os resultados da pesquisa, contará com duas partes organizadas da seguinte forma: primeira parte com a apresentação dos resultados gerais da busca, na sequência o resultado da análise sistemática dos textos completos selecionados no processo; e a segunda parte com a apresentação de aplicativos existentes relacionados com a temática.

\subsection{Análises de Artigos Científicos Relacionados a Sistema de Informação Geográfica para o Turista Deficiente}

Os 278 artigos localizados nas bases IEEE, Web of Science, Science Direct e a entrada manual de artigos (conforme os procedimentos descritos na seção anterior), foram escritos por 935 autores, estão publicados em 144 periódicos, e contam com um somatório de 2149 palavras-chave. A frequência das publicações foi maior no período de 2013 a 2018, sobre o tema SIG para o turista deficiente.

A fase de análise sistemática aplicada na leitura integral dos textos permitiu expandir os conhecimentos sobre o tema da pesquisa. Na sequência os 18 artigos lidos, serão analisados, aplicando a técnica de leitura de estudo ou informativa, que segundo Lakatos; Marconi (2003), visa à coleta de informações para determinado propósito, no qual se aplicou as fases de reconhecimento ou prévia, para verificar a relação com o assunto de interesse da pesquisa, a fase exploratória ou pré-leitura e a fase seletiva, que consiste da seleção das informações mais importantes relacionadas com o problema em questão.

Na leitura dos artigos foram identificados os itens: Introdução, os títulos das seções, tabelas e gráficos, definições de teoremas, conclusões e referências. Em sua maioria, os artigos estão escritos em língua inglesa, nos quais se aplicou a tradução livre para confecção da análise.

Os artigos foram separados por duas categorias: Categoria 1 - Tecnologias aplicadas ao turismo em geral; e Categoria 2 - Tecnologias aplicadas ao turista com deficiência. Neste artigo somente serão descritas as análises dos artigos da categoria 2 , por se tratar do turista deficiente, foco da pesquisa.

\subsubsection{Tecnologias Aplicadas ao Turismo em Geral}

Nesta seção estão agrupados os artigos no qual os autores não citam explicitamente ou diretamente a aplicação das tecnologias às pessoas com deficiência. O Quadro 1 na sequencia apresenta os artigos desta categoria, autores, título, ano e número de citações. O número de citações foi obtido utilizando a contagem de citações presentes na pesquisa da ferramenta de busca Google Scholar, pesquisado no dia 30 de julho de 2018. 
Quadro 1: Tecnologias aplicadas ao turismo em geral

\begin{tabular}{|c|c|c|c|}
\hline AUTORES & TÍTULO & ANO & CITAÇÕES \\
\hline (GAVALAS et al., 2014) & Mobile recommender systems in tourism & 2014 & 264 \\
\hline (HUI-WEI et al., 2015) & $\begin{array}{l}\text { Resource navigation system of typical lijiang river } \\
\text { tourism based on GIS and panorama technique }\end{array}$ & 2015 & - \\
\hline (SMIRNOV et al., 2016) & $\begin{array}{l}\text { Application for e-Tourism: Intelligent Mobile Tourist } \\
\text { Guide }\end{array}$ & 2016 & 7 \\
\hline $\begin{array}{l}\text { (BROVELLI; MINGHINI; } \\
\text { ZAMBONI, 2016) }\end{array}$ & Public participation in GIS via mobile applications & 2016 & 43 \\
\hline $\begin{array}{l}\text { (VEENENDAAL; } \\
\text { BROVELLI; WU, 2016) }\end{array}$ & $\begin{array}{l}\text { Cloud/web mapping and geoprocessing services - } \\
\text { Intelligently linking geoinformation }\end{array}$ & 2016 & 3 \\
\hline (RODA et al., 2017) & $\begin{array}{l}\text { An integrated methodology model for smart mobility } \\
\text { system applied to sustainable tourism }\end{array}$ & 2017 & - \\
\hline $\begin{array}{l}\text { (ALI; SAEED; FAGEERI, } \\
\text { 2017) }\end{array}$ & $\begin{array}{l}\text { Web-based GIS Business Hotels Tourism Sites in } \\
\text { Khartoum, Sudan }\end{array}$ & 2017 & 1 \\
\hline (WANCHUN, 2017) & $\begin{array}{l}\text { Design and Implementation of Intelligent Tourism } \\
\text { Platform for Mobile Terminal }\end{array}$ & 2017 & - \\
\hline (XIAO et al., 2018) & $\begin{array}{l}\text { Geoinformatics for the conservation and promotion of } \\
\text { cultural heritage in support of the UN Sustainable } \\
\text { Development Goals }\end{array}$ & 2018 & 2 \\
\hline
\end{tabular}

\subsubsection{Tecnologias Aplicadas ao Turista com Deficiência}

Nesta seção constam os artigos que aplicam tecnologias para apoiar o turista com deficiência, que compreendem as pessoas com deficiência, mobilidade reduzida, idosos, entre outros. As análises versarão sobre os itens: objetivo do artigo; a metodologia utilizada; público alvo; se houve o desenvolvimento ou análise de um aplicativo; o local onde foi aplicado ou testado o aplicativo; e resultados e conclusões. O Quadro 2 apresenta os artigos, autores, título, ano e número de citações. O número de citações foi obtido utilizando a contagem de citações presentes na pesquisa da ferramenta de busca Google Scholar, pesquisado no dia 30 de julho de 2018.

Quadro 2: Tecnologias aplicadas ao turista com deficiência

\begin{tabular}{|c|c|c|c|}
\hline AUTORES & TÍTULO & ANO & CITAÇÕES \\
\hline $\begin{array}{l}\text { (RODRIGUEZ-SANCHEZ } \\
\text { et al., 2014) }\end{array}$ & $\begin{array}{l}\text { Accessible smartphones for blind users: A case study for a } \\
\text { wayfinding system }\end{array}$ & 2014 & 30 \\
\hline (KULAKOV et al., 2014) & $\begin{array}{l}\text { Accessibility Map and "Social navigator" services for } \\
\text { persons with disabilities }\end{array}$ & 2014 & 8 \\
\hline (SIGNORETTI et al., 2015) & $\begin{array}{l}\text { Trip } 4 \text { All: A Gamified App to Provide a New Way to Elderly } \\
\text { People to Travel }\end{array}$ & 2015 & 7 \\
\hline $\begin{array}{l}\text { (KULAKOV; SHABAEV; } \\
\text { SHABALINA, 2015) }\end{array}$ & $\begin{array}{l}\text { The route planning services approach for people with } \\
\text { disability }\end{array}$ & 2015 & 5 \\
\hline $\begin{array}{l}\text { (GARCÍA; PLAZA; } \\
\text { JIMÉNEZ, 2016) }\end{array}$ & $\begin{array}{l}\text { Metodología para el análisis de la continuidad del espacio } \\
\text { público para el turista con movilidad reducida }\end{array}$ & 2016 & - \\
\hline (KOZIEVITCH et al., 2016) & $\begin{array}{l}\text { An Alternative and Smarter Route Planner for Wheelchair } \\
\text { Users - Exploring Open Data }\end{array}$ & 2016 & 5 \\
\hline $\begin{array}{l}\text { (MOBASHERI; DEISTER; } \\
\text { DIETERICH, 2017) }\end{array}$ & $\begin{array}{l}\text { Wheelmap: the wheelchair accessibility crowdsourcing } \\
\text { platform }\end{array}$ & 2017 & 3 \\
\hline (SANTOS et al., 2017) & $\begin{array}{l}\text { Using POI functionality and accessibility levels for } \\
\text { delivering personalized tourism recommendations }\end{array}$ & 2017 & - \\
\hline (TONGKAW, 2017) & GIS Application Management for Disabled People & 2017 & - \\
\hline
\end{tabular}
Fonte: Elaborado pelos autores (2018).

A seguir serão descritas as análises dos artigos apresentados no Quadro 2, que trata sobre as tecnologias aplicadas ao turista com deficiência, foco da presente pesquisa.

Os autores Rodriguez-Sanchez et al. (2014) iniciam o artigo citando questões sobre acessibilidade e mobilidade, pessoa com deficiência no turismo e suas necessidades. O objetivo do artigo está focado em sistemas de orientação para usuários com problemas de visão, abordando as dificuldades e necessidade de assistência. O enfoque do artigo é a criação de um aplicativo de orientação adaptável para smartphones, acessível, para auxiliar usuários com problemas de cegueira. $\mathrm{O}$ artigo apresenta um estudo de tecnologias, 
teste de software de leitura de tela em Android, testes com usuários e estrutura da aplicação. O usuário pode escolher pontos de interesse no qual o aplicativo irá orientar o usuário por meio do GPS e funções da aplicação.

O artigo de Rodriguez-Sanchez et al. (2014) apresenta a pesquisa realizada na universidade Rey Juan Carlos, Madrid, Espanha. Foram relatados os testes, feedback dos usuários e resultados. No decorrer da pesquisa, os autores citam avanços nas tecnologias para smartphones e destaca que melhorias devem ser implementadas em interfaces touch-screen e comandos de voz. O artigo descreve que o aplicativo desenvolvido auxiliou as pessoas a realizar suas tarefas, que este aplicativo pode ser utilizado por muitas pessoas, independente de deficiência. Como trabalhos futuros são propostos uma possível integração de um kit de avaliação no aplicativo e estudos para inclusão em atividades laborais de pessoas com deficiência.

No artigo de Kulakov et al. (2014), os autores descrevem que o trabalho é parte de um grande projeto relacionado ao desenvolvimento de informações para pessoas com deficiência. Discorre sobre cidades na Rússia que não propiciam à acessibilidade, e cita que o trabalho irá abordar os itens: servidor web de serviços, um aplicativo móvel e o serviço de planejamento de rotas. Os autores descrevem no artigo o desenvolvimento de um aplicativo com informações de acessibilidade, no qual são apresentados pontos em um mapa, no qual os pontos podem ser filtrados por três categorias, que são: atividades organizacionais; tipos de deficiência; e níveis de acessibilidade.

A arquitetura do sistema para informações de acessibilidade, que envolve o servidor web de serviços, um aplicativo móvel e o serviço de planejamento de rotas, destacado no artigo de Kulakov et al. (2014), engloba as tecnologias: MySQL, JSON, WebAPI module, HTML 5, JavaScript, CSS3, JQuery, JQuery Mobile, AngularJS, PhoneGap, ASP.NET MVC4, OpenStreetMap. No serviço de planejamento de rotas, denominado "Navegador Social" são apresentadas funções do serviço, algoritmo de estimativa de acessibilidade de rotas, a classificações de usuários e as abordagens de visualização de obstáculos. O artigo apresentou uma infraestrutura de informação, que contém vários serviços móveis e web. O servidor web de serviços, denominado "Passaportes de Acessibilidade" foi colocado em funcionamento pelo Ministério da Saúde e Desenvolvimento Social da Carélia, Rússia. Concluindo o artigo, os autores comentam que o banco de dados de serviços contém informações sobre 450 objetos socialmente significativos cadastrados (pontos de interesse, atrações turísticas, entre outros) com coordenadas geográficas e níveis de acessibilidade.

Signoretti et al. (2015) apresenta uma aplicação que realiza interação com o turista em formato de jogo, sendo o público alvo os turistas idosos, utilizando a geolocalização e mapas hierárquicos georreferenciados, no qual o turista interage com o jogo, com informações históricas, texto, som, imagem e vídeo, bem como relacionados a desafios. $\mathrm{O}$ artigo conceitua o turista sênior, experiência de viagem e destaca porque a gamificação é vista como importante para a motivação das pessoas. Os autores citam as fases de desenvolvimento que envolveu o levantamento de requisitos, a criação do protótipo e a avalição do aplicativo.

A estrutura do aplicativo descrito no artigo de Signoretti et al. (2015) possui um mapa base, sub-mapa, área de interesse e pontos de interesse. O protótipo inicial foi desenvolvido para Windows Phone 8.1 usando Visual Studio 2013 IDE. Os testes do protótipo utilizaram o mapa do campus da Universidade de Aveiro, Portugal. Os autores citam que o aplicativo está em fase inicial e os primeiros resultados fortalecem o atingimento dos objetivos do protótipo. Citam o estabelecimento da Plataforma de Gamificação Georreferenciada e que os próximos passos é o desenvolvimento de um roteiro para o centro histórico da cidade.

Kulakov; Shabaev; Shabalina (2015) descrevem que a pesquisa apresentada é parte de um projeto maior relacionado ao desenvolvimento de informações para pessoas com deficiência. $\mathrm{O}$ trabalho trata-se da extensão do artigo destacado de Kulakov, Apanasik, Shabaev e Shabalina (2014) que aborda um sistema composto por um servidor web de serviços, um aplicativo móvel e o serviço de planejamento de rotas para pessoas com deficiência.

Kulakov; Shabaev; Shabalina (2015) apresentam uma revisão de bibliotecas e tecnologias que são usadas para soluções de navegação e planejamento de rotas. Cita as tecnologias Google Directions API, JavaScript API Yandex.Map, e Open Source Routing Machine (OSRM). Na sequência, descreve o serviço móvel "Navegador social", sua arquitetura, algoritmos e funções, métodos desenvolvidos de navegação para pessoas com deficiência; destaca as funções do serviço de planejamento de rotas denominado "Navegador Social", que compõe uma coleção de dados, o planejamento e compartilhamento de rota, e assistência de áudio. Os autores descrevem a implementação do serviço "Navegador Social", a interface do usuário e demonstram o uso prático. Os autores concluem dizendo que o trabalho apresentado é uma parte da 
infraestrutura desenvolvida que contém vários serviços móveis e da web. O serviço foi oferecido a voluntários e pessoas com deficiência, mas não comenta o feedback destes usuários.

García; Plaza; Jiménez (2016) tem como objetivo desenvolver uma metodologia para avaliar e categorizar o nível de acessibilidade do espaço público de uma cidade para auxiliar os cidadãos e turistas. O artigo aborda a dificuldade em obter informações de locais para as pessoas com deficiência na web. Destaca projetos e pesquisas na área de mobilidade, bem como cita o aplicativo Wheelmap, entre outros. Na metodologia, classifica o nível de acessibilidade em interesse físico e social, cita barreiras arquitetônicas e ilustra as informações com mapas. Na conclusão do artigo, os autores citam que a pessoa com deficiência tem a necessidade de conhecer o nível de acessibilidade e se planejar, e que a metodologia serve como ponto de partida para outras pesquisas no assunto.

A pesquisa de Kozievitch et al. (2016), preocupa-se com problemas de rotas e acessibilidade de cadeirantes, no qual é proposto uma metodologia, utilizando dados abertos, de melhores rotas para os cadeirantes. Os autores citam que existe uma falta de informações sobre acessibilidade em espaços urbanos, e que isso contribui para que a maioria dos estudos sobre rotas acessíveis estão restritos a cidades ou distritos. Cita trabalhos relacionados, e a iniciativa do projeto Wheelmap, que trabalha com a coleta de dados de pontos de interesse.

O método descrito no artigo de Kozievitch et al. (2016) utiliza mapas de base para criar mapas derivados com informações mais detalhadas, rotas, rampas, obstruções, para auxiliar nas decisões de locomoção. O estudo de caso foi aplicado na cidade de Curitiba / PR, utilizando aquisição de dados do Instituto de Pesquisa e Planejamento Urbano de Curitiba, e do OpenStreetMap. Tecnologias utilizadas: PostGIS e QGIS. O artigo apresenta conceitos e a aplicação de um planejador de rotas para usuários cadeirantes, e finaliza com trabalhos futuros, a aplicação da tecnologia na região metropolitana de Curitiba, a personalização de pesos para diferentes usuários e a integração do modo off-line.

No artigo de Mobasheri; Deister; Dieterich (2017) os autores iniciam discorrendo sobre o aplicativo Wheelmap, que é um mapa de acessibilidade para cadeirantes, baseado no banco de dados do OpenStreetMap, no qual usuários provêm informação para outros usuários sobre como o local em que estão está acessível. O artigo discorre sobre a participação voluntária para o mapeamento de dados de acessibilidade, e como foram implementadas a arquitetura, as funcionalidades e as tecnologias. Tecnologias utilizadas no aplicativo Wheelmap: Ruby on Rails, Rosemary, OpenStreetMap API, Maki, MapBox, ArcGIS. $\mathrm{O}$ artigo cita que o aplicativo é compatível com dispositivos Android e iPhone. Os autores concluem citando que o aplicativo Wheelmap tem ganhado grande interesse e atenção das comunidades relacionadas à acessibilidade, sua base de dados possui mais de 800.000 pontos de interesse, o projeto foi executado por um time de uma organização não governamental chamada Sozialhelden, e que o aplicativo têm ganhado muitos prêmios nacionais e internacionais.

Santos et al. (2017) abordam perspectivas sobre o turismo, pessoas com deficiência e pontos de interesse. O objetivo principal da pesquisa é o desenvolvimento de um sistema de recomendação em relação a um conjunto de questões físicas e cognitivas. Os pontos de interesse, presentes no sistema, foram modelados com foco em limitações físicas, psicológicas e em nível de acessibilidade. Os autores citam exemplos de outros sistemas desenvolvidos para recomendação na área de turismo como: Personal Travel Assistant (PTA), CAPA, MultiMundus, TIP, Heracles, Proximo, GeoNotes, MacauMap, EtPlanner, mobiDENK, Cyberguide, CATIS, Deep Map, Tousplan project, Tours Planning Support System, ITravel, 3D-GIS Hybrid, POST-VIA360, TRSO project, ETourism2.0.

Santos et al. (2017) citam que utilizaram informações do site de governamental de turismo de Portugal para o desenvolvimento do sistema de recomendação. A metodologia é baseada no conhecimento de informações dos usuários, dos pontos de interesse e algoritmos propostos para atuar na recomendação. $\mathrm{O}$ protótipo foi implementado em PHP e JavaScript e testado com usuários. Os autores concluem o artigo demonstrando que a contribuição da pesquisa foi mostrar funcionalidades do sistema, os perfis de usuário, e as recomendações que o turista pode fazer quando está visitando um ponto turístico. Como trabalho futuro, os autores destacam a aquisição automatizada de fontes de informações e sua classificação.

Tongkaw (2017) relata que o objetivo da pesquisa é desenvolver e projetar um SIG para apoiar as pessoas com deficiência. O sistema foi projetado para coletar o estado atual das pessoas com deficiência e apresentar informações sobre os tipos de deficiência e número de pessoas nesta condição no Google Maps. O método de pesquisa desta as fases de projeto do sistema, análise do sistema, desenvolvimento, teste, implementação e manutenção. A pesquisa destacou que o sistema deve ter duas interfaces, de acordo com o perfil do usuário, que pode ser administrador ou usuário. As tecnologias utilizadas foram Java e PHP. Com resultados do trabalho, o autor destaca que a pesquisa trabalhou em conjunto com o Ministério do Desenvolvimento Social 
e Segurança Humana da Tailândia, para coletar informações e testar o aplicativo, também cita como trabalhos futuros agregar as funções de guia de áudio na navegação e sugestão de rotas.

É importante salientar que os artigos de Rodriguez-Sanchez et al. (2014) e Signoretti et al. (2015) abordaram o deficiente visual e o turista sênior (idoso), apresentando um aplicativo de orientação para cegos e um aplicativo em formato de jogo, ambos para smartphones, destacando que os aplicativos foram testados para analisar as funcionalidades e a experiência dos usuários.

De uma forma geral, os artigos de: Kulakov et al. (2014), Kulakov; Shabaev; Shabalina (2015), García; Plaza; Jiménez (2016), Kozievitch et al. (2016), Mobasheri; Deister; Dieterich (2017), Santos et al. (2017) e Tongkaw (2017) apresentam aplicativos em que o público alvo são as pessoas com deficiência, com foco no deficiente físico. Alguns artigos citam o cadeirante e seus problemas relacionados à mobilidade e a acessibilidade em ambientes públicos e turísticos. Sobre este tema, aplicativos que podem auxiliar o turista deficiente cadeirante, se trata a próxima seção deste artigo.

\subsection{Levantamento de Aplicativos Existentes}

Os artigos selecionados na pesquisa apresentaram várias soluções, sistemas e aplicativos que podem auxiliar o turista deficiente em relação à acessibilidade. Além das tecnologias já apresentadas na revisão sistemática, buscou-se identificar aplicativos mais utilizados pelos usuários e que tem relação com a temática de acessibilidade no turismo. Foram encontrados dois aplicativos, o Wheelmap e o Guia de Rodas, buscandose no serviço de distribuição digital de aplicativos Google Play Store.

Os aplicativos se destacam por apresentar um número superior de mais de 10.000 instalações, o que indica uma grande comunidade de usuários; e por estarem relacionados ao tema acessibilidade e nas categorias Mapas e navegação; e Turismo e local, de acordo com a plataforma Play Store do Google, em julho de 2018.

A seguir serão apresentados estes dois aplicativos, um europeu e outro de procedência brasileira, que são exemplos de aplicativos que podem ajudar as pessoas com deficiência, mais especificamente o deficiente físico cadeirante. Serão demonstrados os itens: Aplicativo, Sistema Operacional, Desenvolvedor, Objetivo, Idioma e Funções.

Segundo a norma ABNT NBR ISO 9241-11:2011, que trata sobre requisitos ergonômicos para o trabalho com dispositivos de interação visual e orientações sobre usabilidade, seção contexto de uso, que descreve caraterísticas relevantes dos usuários, tarefas, equipamento e o ambiente físico e social nos quais um produto é usado; os itens de avaliação dos aplicativos Guia de Rodas e Wheelmap estão baseados na referida norma e foram escolhidos para facilitar o entendimento sobre a avaliação.

Aplicativo: WheelMap (Figura 2). Sistema Operacional: Web, Android, iOS. Desenvolvedor: Sozialhelden e.V., Projeto WheelMap.org. Objetivo: Qualquer pessoa pode pesquisar e adicionar locais públicos no mapa e ranquear quão acessível está o local. O mapa foi criado em 2010 para ajudar pessoas que utilizam cadeiras de rodas manuais ou automáticas planejarem suas atividades diárias de forma mais fácil.

Idiomas: Alemão, Inglês, Português. Funções: Camada Base: OpenStreetMap; Permite pesquisa de localidade; Função proximidades que apresenta pontos e locais próximos; Filtro por categoria de local; Apresenta os pontos no mapa com as informações do local mapeadas; Os pontos apresentam ícones com a simbologia do local, cores conforme a legenda de acessibilidade: Sim (verde), Limitado (laranja), Não (vermelho), Desconhecido (cinza); Ao clicar em um ponto apresenta o status (Acessível a cadeirantes, Parcialmente acessível a cadeira de rodas, Não acessível para cadeira de rodas, Status desconhecido) e status do banheiro (Banheiro acessível a cadeirantes, Banheiro não é acessível a cadeirantes, Status do banheiro desconhecido); Para alterar/cadastrar uma alguma informação deve-se efetuar login utilizando uma conta do OpenStreetMap (OSM); Pode-se identificar no mapa que um grande conjunto de informações está concentrado na Europa; Em testes na cidade de Foz do Iguaçu/PR, as informações apresentam status "Desconhecido"; Acredita-se que os dados e os pontos são cadastrados diretamente no servidor de mapas OpenStreetMap.

Aplicativo: Guia de Rodas (Figura 3). Sistema Operacional: Android, iOS. Desenvolvedor: Bruno Mahfuz. Objetivo: Aplicativo colaborativo para avaliar e consultar a acessibilidade de estabelecimentos. Com o objetivo de facilitar a vida das pessoas, fazer avaliação de acessibilidade pelos usuários, utiliza base de dados do Foursquare. Idiomas: Português e Espanhol. Funções: Utiliza a base do Foursquare para identificar os estabelecimentos. Não apresenta um mapa na tela inicial, permite pesquisar por nome ou selecionar em uma lista de estabelecimentos. É apresentada uma sinalização em cores do nível de acessibilidade: Cinza (não avaliado), Verde (Acessível), Amarelo (Parcialmente acessível) e Vermelho (Não 
acessível). Identificado o estabelecimento, apresenta o status de acessibilidade, sendo possível alterar para as opções: Acessível para pessoas com dificuldade de locomoção; Parcialmente acessível para pessoas com dificuldade de locomoção; Não acessível para pessoas com dificuldade de locomoção. Ao selecionar um estabelecimento, no detalhamento é apresentado: Informações gerais do estabelecimento; Pequeno mapa com o ponto do local; Pode ser categorizado o status de acessibilidade em: Estacionamento, Entrada. Podem ser inseridos comentários relacionados ao estabelecimento.
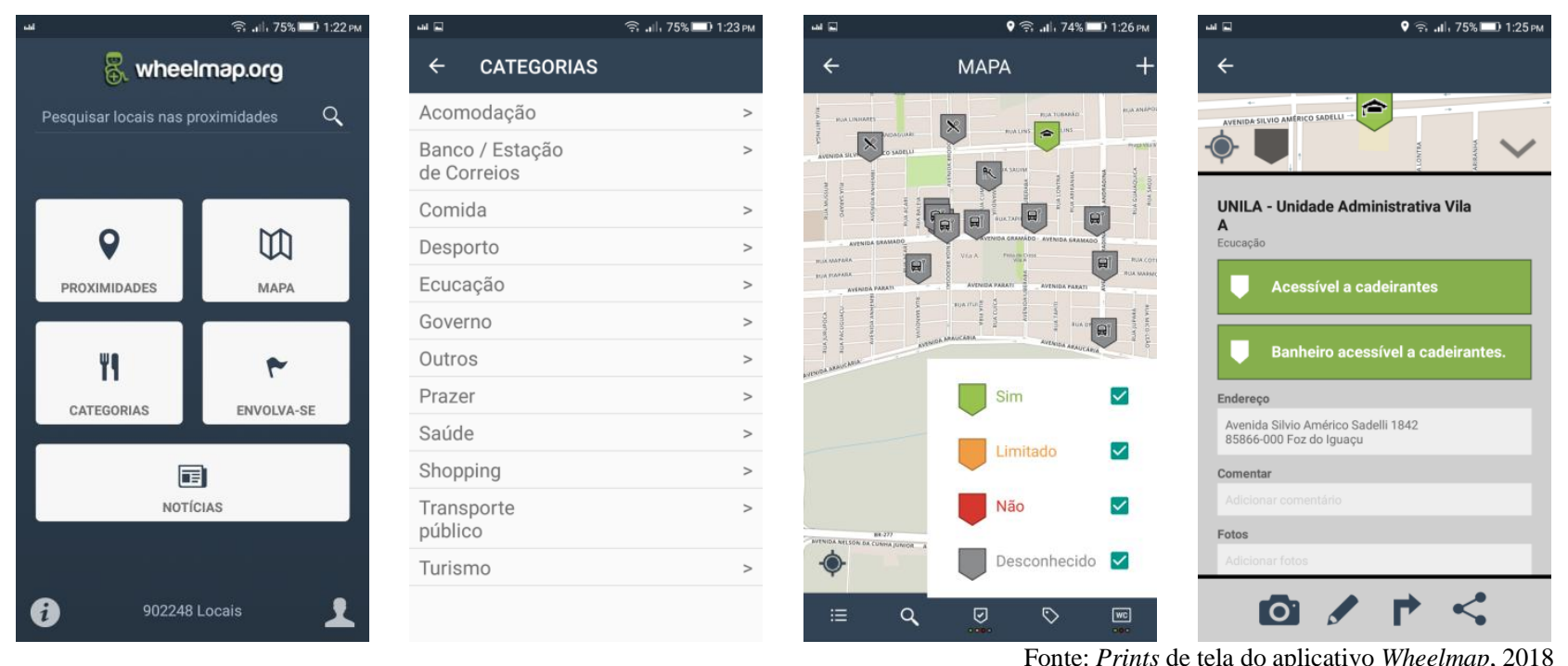

Figura 2: Telas do aplicativo Wheelmap. Notas: $1^{\mathrm{a}}$ ) Tela de abertura do aplicativo; $2^{\mathrm{a}}$ ) Tela com as categorias em que estão agrupados os pontos; $3^{\mathrm{a}}$ ) Tela com o mapa e os pontos, sinalizados por cores; $4^{\mathrm{a}}$ ) Tela que apresenta em detalhe um ponto cadastrado no aplicativo.
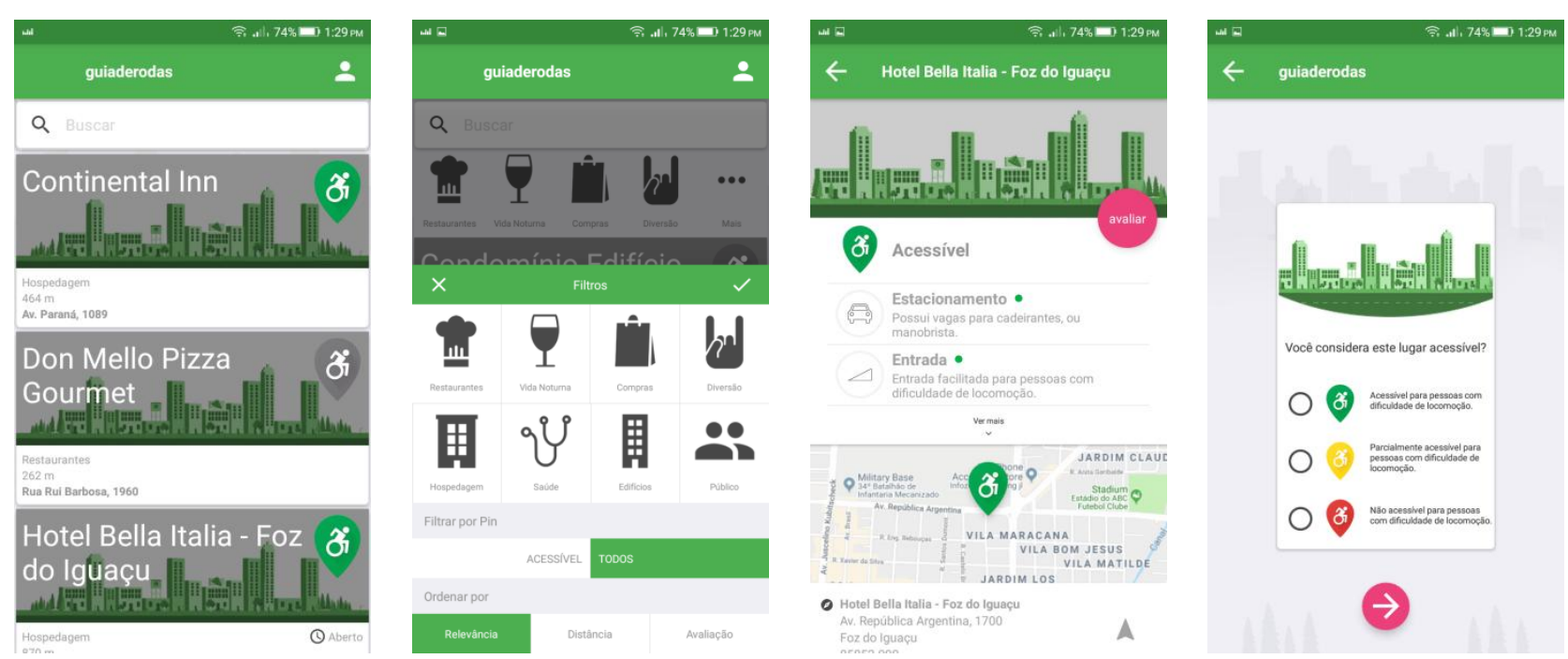

Figura 3: Telas do aplicativo Guia de Rodas. Notas: $1^{\text {a }}$ ) Tela inicial do aplicativo; $2^{\mathrm{a}}$ ) Tela com os possíveis filtros dos tipos de estabelecimento; $3^{\mathrm{a}}$ ) Tela com um estabelecimento selecionado; $4^{\mathrm{a}}$ ) Tela para avaliação de um estabelecimento em relação à acessibilidade do local.

\section{Considerações Finais}

O presente estudo teve o propósito de analisar artigos científicos relacionados à SIG para o turista deficiente e buscar aplicativos existentes, utilizando uma revisão sistemática de artigos dos últimos cinco anos e o destaque de dois aplicativos para o deficiente físico cadeirante. No decorrer da pesquisa constatouse que no Brasil o tema ainda é pouco explorado, mas que em nível internacional o tema é bem discutido e aplicado em estudos de casos, geralmente restritos a cidade e a distritos. As tecnologias levantadas, em sua maioria, são tecnologias livres, em que é possível desenvolver projetos inovadores com baixo custo de execução. 
Nos artigos apresentados, verificou-se que, em sua maioria, existe a necessidade de um levantamento de requisitos, uma definição de uma arquitetura do sistema, composição de tecnologias, interfaces de usuário e administrador, testes do sistema no ambiente proposto e análises de utilização. Acredita-se que estes passos são fundamentais para o desenvolvimento de um projeto bem estruturado de um aplicativo ou sistema, no qual devem estar presentes as especificidades a serem consideradas em relação às pessoas com deficiência e mobilidade reduzida.

A pesquisa não teve a intenção de esgotar sobre o assunto apresentado neste artigo, mas espera-se que os resultados colaborem para disseminação e desenvolvimento de novas tecnologias e projetos no âmbito de sistemas de informação geográfica aplicado as pessoas com deficiência, tanto no turismo, quanto em outras áreas.

Segundo revela a pesquisa de demanda internacional do Ministério do Turismo, em 2016, Foz do Iguaçu ocupou o terceiro lugar na lista dos dez municípios brasileiros mais visitados a lazer, atrás do Rio de Janeiro e Florianópolis (PARANÁ, 2018). Neste contexto, como trabalhos futuros, o autor tem como propósito o pesquisar e desenvolver um aplicativo com informações de acessibilidade para auxiliar na mobilidade das pessoas com deficiência na cidade turística de Foz do Iguaçu/PR.

\section{Referências}

ABNT. NBR ISO 9241-11:2011. Requisitos ergonômicos para o trabalho com dispositivos de interação visual. Parte 11: Orientações sobre usabilidade. Rio de Janeiro: Associação Brasileira de Normas, 2011.

ALI, T. A. T.; SAEED, R. A.; FAGEERI, S. O. Web-based GIS Business Hotels Tourism Sites in Khartoum, Sudan. Proceedings - 2017 International Conference on Communication, Control, Computing and Electronics Engineering, ICCCCEE 2017, 2017.

BRASIL. Decreto $n^{0} 6.949$ de 25 de agosto de 2009. Promulga a Convenção Internacional sobre os Direitos das Pessoas com Deficiência e seu Protocolo Facultativo, assinados em Nova York, em 30 de março de 2007. Disponível em: <http://www.planalto.gov.br/ccivil_03/_ato20072010/2009/decreto/d6949.htm>. Acesso em: 15 fev. 2019.

BROVELLI, M. A.; MINGHINI, M.; ZAMBONI, G. Public participation in GIS via mobile applications. ISPRS Journal of Photogrammetry and Remote Sensing, v. 114, p. 306-315, 2016.

DARCY, S.; CAMERON, B.; PEGG, S. Accessible tourism and sustainability: A discussion and case study. Journal of Sustainable Tourism, v. 18, n. 4, p. 515-537, 2010.

DARCY, S.; DICKSON, T. J. A whole-of-life approach to tourism: The case for accessible tourism experiences. Journal of Hospitality and Tourism Management, v. 16, n. 1, p. 32-44, 2009.

GARCÍA, J. J. C.; PLAZA, A. G.; JIMÉNEZ, C. R. METODOLOGÍA PARA EL ANÁLISIS DE LA CONTINUIDAD DEL ESPACIO PÚBLICO PARA EL TURISTA CON MOVILIDAD REDUCIDA. Gran Tour: Revista de Investigaciones Turísticas, p. 132-148, 2016.

GAVALAS, D. et al. Mobile recommender systems in tourism. Journal of Network and Computer Applications, v. 39, n. 1, p. 319-333, 2014.

HUI-WEI, S. et al. Resource navigation system of typical lijiang river tourism based on GIS and panorama technique. Proceedings - 7th International Conference on Intelligent Computation Technology and Automation, ICICTA 2014, v. 4, n. 978, p. 609-615, 2015.

IBGE. Censo demográfico : 2010 : características gerais da população, religião e pessoas com deficiência. Disponível em: <https://biblioteca.ibge.gov.br/visualizacao/periodicos/94/cd_2010_religiao_deficiencia.pdf〉. Acesso em: 5 dez. 2019.

KOZIEVITCH, N. P. et al. An Alternative and Smarter Route Planner for Wheelchair Users - Exploring Open Data. Proceedings of the 5th International Conference on Smart Cities and Green ICT Systems, p. 94-99, 2016.

KULAKOV, K. A. et al. Accessibility Map and "Social navigator" services for persons with disabilities. Conference of Open Innovation Association, FRUCT, p. 69-76, 2014.

KULAKOV, K. A.; SHABAEV, A. I.; SHABALINA, I. M. The route planning services approach for people with disability. 2015 17th Conference of Open Innovations Association (FRUCT). Anais...IEEE, 
abr. 2015Disponível em: 〈http://ieeexplore.ieee.org/document/7117977/>

LAKATOS, E. M.; MARCONI, E. M. Fundamentos de metodologia científica. São Paulo: Editora Atlas, 2003.

LONGLEY, P. A. et al. Sistemas e Ciência da Informação Geográfica. 3. ed. Porto Alegre: Bookman, 2013.

MOBASHERI, A.; DEISTER, J.; DIETERICH, H. Wheelmap: the wheelchair accessibility crowdsourcing platform. Open Geospatial Data, Software and Standards, v. 2, n. 1, p. 27, 2017.

PARANÁ. Turismo em Números 2018. Disponível em: <http://www.turismo.pr.gov.br/arquivos/File/TurismoemNumeros2018_1.pdf>. Acesso em: 28 fev. 2019.

RODA, M. et al. An integrated methodology model for smart mobility system applied to sustainable tourism. 2017 IEEE 3rd International Forum on Research and Technologies for Society and Industry (RTSI), p. 1-6, 2017.

RODRIGUEZ-SANCHEZ, M. C. et al. Accessible smartphones for blind users: A case study for a wayfinding system. Expert Systems with Applications, v. 41, n. 16, p. 7210-7222, 2014.

SANTOS, F. et al. Using POI functionality and accessibility levels for delivering personalized tourism recommendations. Computers, Environment and Urban Systems, 2017.

SIGNORETTI, A. et al. Trip 4 All: A Gamified App to Provide a New Way to Elderly People to Travel. Procedia Computer Science, v. 67, n. Dsai, p. 301-311, 2015.

SMIRNOV, A. et al. Application for e-Tourism: Intelligent Mobile Tourist Guide. Proceedings - 2015 IIAI 4th International Congress on Advanced Applied Informatics, IIAI-AAI 2015, p. 40-45, 2016.

TONGKAW, S. GIS Application Management for Disabled People. IOP Conference Series: Materials Science and Engineering, v. 226, p. 012112, 2017.

TRANFIELD, D.; DENYER, D.; SMART, P. Towards a Methodology for Developing Evidence-Informed Management Knowledge by Means of Systematic Review. British Journal of Management, v. 14, n. 3, p. 207-222, set. 2003.

UNWTO. Global Code of Ethics for Tourism - Article 7. Disponível em: <http://ethics.unwto.org/en/content/global-code-ethics-tourism-article-7>. Acesso em: 1 ago. 2018.

VEENENDAAL, B.; BROVELLI, M. A.; WU, L. Cloud/web mapping and geoprocessing services Intelligently linking geoinformation. ISPRS Journal of Photogrammetry and Remote Sensing, v. 114, p. 243-244, 2016.

WANCHUN, Z. Design and Implementation of Intelligent Tourism Platform for Mobile Terminal. 2017 International Conference on Smart Grid and Electrical Automation (ICSGEA), p. 705-708, 2017.

WTTC. Travel \& Tourism Economic Impact 2018. London: [s.n.]. Disponível em: <https://www.wttc.org/-/media/files/reports/economic-impact-research/countries-2018/brazil2018.pdf>.

XIAO, W. et al. Geoinformatics for the conservation and promotion of cultural heritage in support of the UN Sustainable Development Goals. ISPRS Journal of Photogrammetry and Remote Sensing, v. 142, p. 389-406, 2018. 